\title{
Persistent homology-based gait recognition robust to upper body variations
}

\author{
Javier Lamar-Leon ${ }^{1}$, Raul Alonso-Baryolo ${ }^{2}$, Edel Garcia-Reyes ${ }^{3}$, Rocio Gonzalez-Diaz ${ }^{4}$ \\ ${ }^{1,2,3}$ Pattern Recognition Department, Advanced Technologies Application Center, Havana, Cuba. \\ ${ }^{4}$ Applied Math Dept, School of Computer Engineering, University of Seville, Seville, Spain. \\ ${ }^{1}$ jlamar@cenatav.co.cu, ${ }^{2}$ rbaryolo@cenatav.co.cu, ${ }^{3}$ egarcia@cenatav.co.cu, ${ }^{4}$ rogodi@us.es
}

\begin{abstract}
Gait recognition is nowadays an important biometric technique for video surveillance tasks, due to the advantage of using it at distance. However, when the upper body movements are unrelated to the natural dynamic of the gait, caused for example by carrying a bag or wearing a coat, the reported results show low accuracy. With the goal of solving this problem, we apply persistent homology to extract topological features from the lowest fourth part of the body silhouettes. To obtain the features, we modify our previous algorithm for gait recognition, to improve its efficacy and robustness to variations in the amount of simplices of the gait complex. We evaluate our approach using the CASIA-B dataset, obtaining a considerable accuracy improvement of $93.8 \%$, achieving at the same time invariance to upper body movements unrelated with the dynamic of the gait.
\end{abstract}

\section{INTRODUCTION}

Persons recognition at distance, without the subject cooperation, is an important task in video surveillance. Very few biometric techniques can be used in these scenarios. Gait recognition is a technique with special attention under these circumstances due to its advantages, since the features can be extracted from any viewpoint and at bigger distances than other biometric approaches.

Currently, there are good results in the state of the art for persons walking under natural conditions (without carrying a bag or wearing a coat). See, for example, [1], [2], [3]. However, it is not common for people to walk without carrying a bag or anything that changes the natural gait. Moreover, people usually perform movements with the upper body part unrelated to the natural dynamic of the gait.

Up to now, the most successful approaches in gait recognition use silhouettes to get the features. Among the silhouettebased techniques, the best results have been obtained from the methods based in Gait Energy Images (GEI) [4], [1], [2]. Besides, the GEI methods have been used to eliminate the effects of carrying a bag or wearing a coat in [5], [2], [1]. Generally, these strategies are affected by a small number of silhouettes (one gait cycle or less). Moreover, the temporal order in which silhouettes appear is not captured in those representations, loosing the relative relations of the movements in time. On the other hand, the features extracted by these methods are highly correlated with errors in the segmentation of the silhouettes [6]. These errors frequently appear in the existing algorithms for background segmentation. This implies that GEI methods are influenced by the shape of the silhouette instead of the relative positions among the parts of the body while walking. The accuracy in gait recognition for persons carrying bag or using coat can be consulted in [1] for the CASIA-B gait dataset. The authors in [1] used features from the full body but the results were not satisfactory: For instance, the best result for persons walking with coats was $32.7 \%$ using lateral view, while the worst result was $24.6 \%$, using frontal view. Besides, for persons carrying a bag, the best result was $80.2 \%$ using frontal view and the worst $52.0 \%$, using lateral view.

In this paper, we concentrate in overcoming most of the difficulties explained above, which takes us to get promising results as we will see later. First, given the fact that humans can only move using the legs in a coordinated fashion, and according to the analysis done before, it would be convenient to use only features obtained from the lower part of the body (i.e., the legs) to identify persons by their gait. Following this idea, in this work, we get a good accuracy using only the legs to extract features, thus avoiding many of the effects arising from the variability in the upper body part. Moreover, anthropocentric studies [7] confirm that the differences between people's gait are in the relative positions among the parts of the body while walking. According to this, in this work, the gait is modeled using a topological representation, since it gives features of the objects that are invariant to deformation. Notice that the human-being while walks can be considered as an object under continuous deformation (i.e., a non-rigid object). Roughly speaking we can say that topology (or more precisely, persistent homology) is used here just as we did in our previous approaches [3], [8], [9], [10] but, as we will see later, there are slight differences in the present method with respect to our previous ones, obtaining in this case a more robust and efficient approach.

Summing up, we start the procedure with a sequence of silhouettes obtained from a video. To decrease the negative effects of variations unrelated to the gait in the upper body part, we only select the lowest fourth part of the body silhouette (legs-silhouette). A simplicial complex $\partial K(I)$ which represents the human gait is then constructed in Section II. Sixteen persistence barcodes (a known tool in the Theory of Persistent Homology), are then computed in Section III 
considering, respectively, the distance to eight fixed planes ( 2 horizontal planes, 2 vertical planes, 2 oblique planes and 2 depth planes) in order to completely capture the movement in the gait sequence. More concretely, for each plane $\pi$, we compute two persistence barcodes: one to detect the variation of connected components and the other to detect the variation of tunnels when we go through $\partial K(I)$ in a direction perpendicular to the plane $\pi$. Putting together all this information, we construct a vector (called topological signature) associated to each gait sequence. To compare two topological signatures, we use the angle between both vectors. Experimental results are showed in Section IV and are analyzed in Section V. Conclusions are finally given in Section VI.

\section{TOPOLOGICAL MODEL OF THE GAIT: SimPliCiAL COMPLEXES}

In this section we introduce the construction of the simplicial complex $\partial K(I)$ which represents the human gait.

As already stated, our goal is to decrease the negative effects of variations unrelated to the gait in the upper body part. This helps to avoid a lot of noise, for example related to hand gestures like talking on cell. For accomplishing this, we select the lowest fourth part of the body silhouette (legssilhouette) (see Fig. 1). This selection is endorsed by the result in [5], which shows that this part of the body provides most of the necessary information for classification.

We start the procedure with a sequence of silhouettes obtained from a video. With the intention of a fair comparison, we get the sequences from the background segmentation provided in CASIA-B dataset ${ }^{1}$.

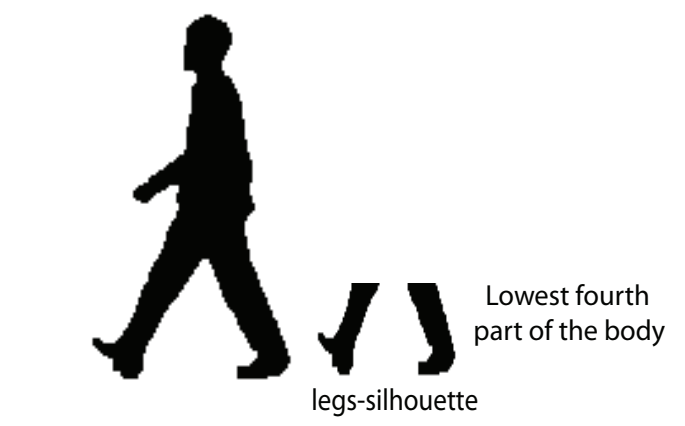

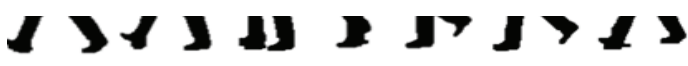 \\ legs-sequence}

Fig. 1. Sequence of gait legs-silhouettes.

As we did in our previous papers, [3], [8], [9], [10], for obtaining the simplicial complex $\partial K(I)$ from a gait, we first build a $3 D$ binary digital image $I=\left(\mathbb{Z}^{3}, B\right)$ by stacking $k$ consecutive silhouettes. A $3 D$ binary digital image is a pair $I=\left(\mathbb{Z}^{3}, B\right)$, where $B \subset \mathbb{Z}^{3}$ is the foreground and $B^{c}=\mathbb{Z}^{3} \backslash B$ the background. Later, $I=\left(\mathbb{Z}^{3}, B\right)$ is used to derive a cubical complex $Q(I)$. The cubical complex $Q(I)$ is a combinatorial structure constituted by a set of

\footnotetext{
${ }^{1}$ http://www.cbsr.ia.ac.cn/GaitDatasetB-silh.zip
}

unit cubes with faces parallel to the coordinate planes and vertices in $\mathbb{Z}^{3}$, together with all its faces. The 0 -faces of a cube $c$ are its 8 corners (vertices), its 1 -faces are its 12 edges, its 2-faces are its 6 squares and, finally, its 3 -face is the cube itself. More concretely, a cube with vertices $V=\{(i, j, k),(i+1, j, k),(i, j+1, k),(i, j, k+1),(i+1, j+$ $1, k),(i+1, j, k+1),(i, j+1, k+1),(i+1, j+1, k+1)\}$, with $(i, j, k) \in \mathbb{Z}^{3}$, is in $Q(I)$ if and only if $V \subseteq B$. Finally, the squares that are faces of exactly one cube in $Q(I)$ are divided into two triangles. These triangles together with their faces (vertices and edges) form the simplicial complex $\partial K(I)$ (see Fig. 2).

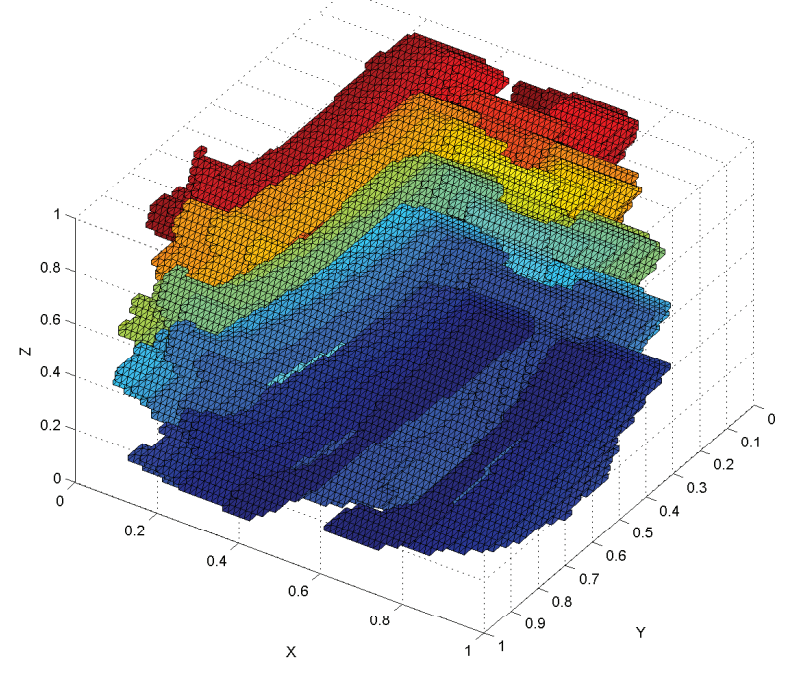

Fig. 2. Simplicial complex $\partial K(I)$ of a legs-sequence. The $x$ axis represents the width of the legs-silhouettes, $y$ axis the height of the legs-silhouettes, while $z$ axis represents the number of silhouettes. All the axis are normalized to $[0,1]$

The next step is to sort the simplices of $\partial K(I)$ in order to obtain a filtration, which is a partial ordering of the simplices of $\partial K(I)$ dictated by a filter function $f: \partial K(I) \rightarrow \mathbb{R}$, satisfying that if a simplex $\sigma$ is a face of another simplex $\sigma^{\prime}$ in $\partial K(I)$ then $f(\sigma) \leq f\left(\sigma^{\prime}\right)$ (i.e., $\sigma$ appears before or at the same time that $\sigma^{\prime}$ in the ordering). In our work, we use eight filtrations obtained from eight planes (see Fig. 3).

For each plane $\pi$, we define the filter function $f_{\pi}$ : $\partial K(I) \rightarrow \mathbb{R}$ which assigns to each vertex of $\partial K(I)$ its distance to the plane $\pi$, and for any other simplex of $\partial K(I)$, the biggest distance of its vertices to $\pi$. Ordering the simplices of $\partial K(I)$ according to the values of $f_{\pi}$, we obtain the filtration $K_{\pi}$ for $\partial K(I)$ associated to the plane $\pi$.

Observe that the way to construct the topological signature here is slightly different to the approach given in [3], [8], [9], [10], since, in those works, the filtration is obtained by adding one simplex at each time (i.e., a total ordering of the simplices is constructed). Nevertheless, in this paper, we add a bunch of simplices to the filtration at each time: all 

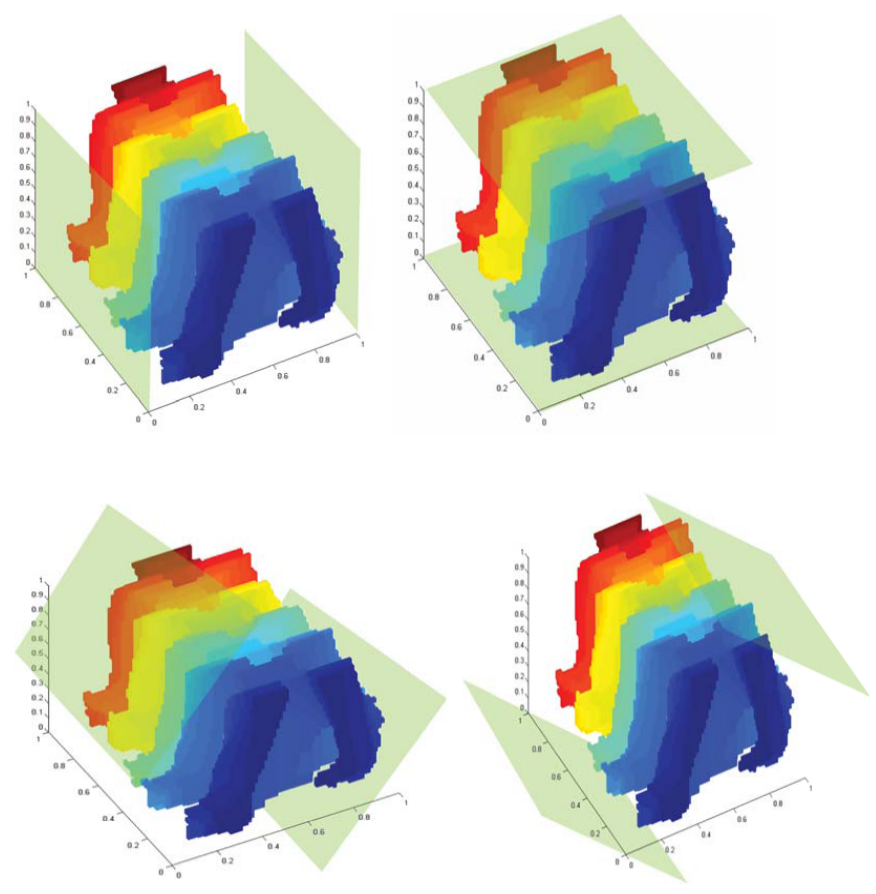

Fig. 3. The eight planes used for computed the eight filtrations for $\partial K(I)$.

the simplices of $\partial K(I)$ with same distance to the reference plane. This way, different times represent sets of simplices with possibly different cardinalities, which makes the method robust to variation in the amount of simplices of the simplicial complex and therefore, robust to noise. Besides, the difficulties we had previously in our papers [3], [8], [9], [10] with the stability of the sorting algorithm disappear in this paper, since each set of simplices in the filtration contains all the simplices with the same distance to a reference plane, and these sets are sorted according to their associated distances.

\section{Persistent homology AND topological SIGNATURE}

Persistent homology is an algebraic tool for measuring topological features of shapes and functions. It is built on top of homology, which is a topological invariant that captures the amount of connected components (or 0-cycles), tunnels (or 1cycles), cavities (or 2-cycles) and similar in higher dimensions of a shape. Small size features in persistent homology are often categorized as noise, while large size features describe topological properties of shapes [11].

To explain the concept of persistent homology, consider a filtration (i.e., a list of sorted simplices) $K_{p}=$ $\left(\sigma_{1}, \sigma_{2}, \ldots, \sigma_{m}\right)$ for a simplicial complex $K$ obtained from a given filter function $f_{p}: K \rightarrow \mathbb{R}$, Suppose that the simplices of the filtration are added in order (i.e., exactly one simplex is added each time). If $\sigma_{i}$ completes a $q$-cycle ( $q$ is the dimension of $\sigma_{i}$ ) when $\sigma_{i}$ is added to $K_{i-1}=\left(\sigma_{1}, \ldots, \sigma_{i-1}\right)$, then a $q$ homology class $\gamma$ is born at time $f_{p}\left(\sigma_{i}\right)$; otherwise, a $(q-1)$ homology class dies at time $f_{p}\left(\sigma_{i}\right)$. The difference between the birth and death times of a homology class is called its
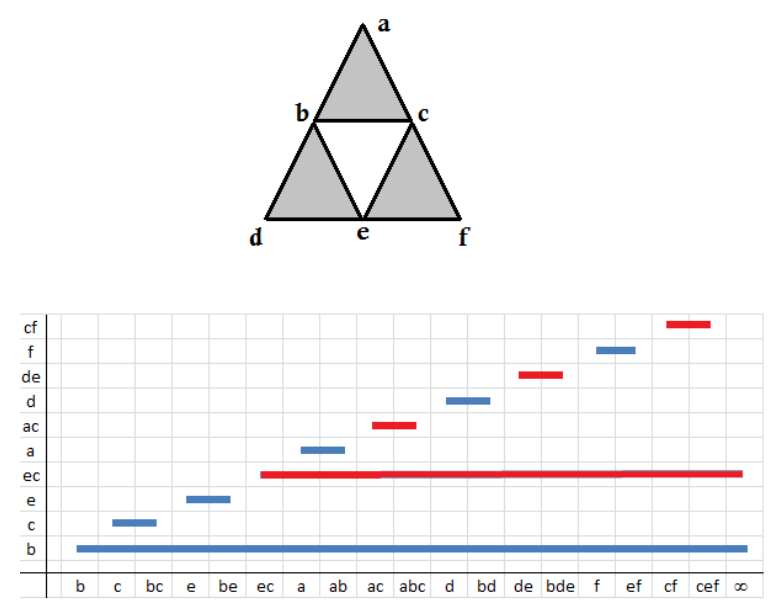

Fig. 4. An example of a persistence barcode obtained from a simplicial complex.

persistence, which quantifies the significance of a topological attribute. If $\gamma$ never dies, we set its persistence to infinity. For a $q$-homology class that is born at time $f_{p}\left(\sigma_{i}\right)$ and dies at time $f_{p}\left(\sigma_{j}\right)$, we draw a segment with endpoints $f_{p}\left(\sigma_{i}\right)$ and $f_{p}\left(\sigma_{j}\right)$ to get the $q$-persistence barcode of the filtration. For a simple example, see Fig. 4, where intervals corresponding to the persistence of 0 -homology classes (i.e. the persistence of connected components) are colored in blue and intervals corresponding to the persistence of 1-homology classes (i.e., the persistence of tunnels) are colored in red. The filtration $F=\{b, c, b c, e, b e, e c, a, a b, a c, a b c, d, b d, d e, b d e$, $f, e f, c f, c e f\}$ which, in this case, is a total ordering, can also be read on the $x$-axis of the diagram. Observe that only two intervals survive until the end (the one corresponding to the connected component and the one corresponding to the tunnel). For a detailed introduction see [11], [12].

In this work, persistence barcodes are first computed for $K_{\pi}$, for each plane $\pi$ shown in Fig. 3. As an example, the persistence barcodes for the video sequences 001-nm-01-090 and 002-nm-01-090 in the CAISA-B dataset are shown in Fig. 5. The red intervals represent the persistence of 0-homology classes while the blue ones represent the persistence of 1homology classes. For computing these barcodes, we used the leftmost plane of Fig. 3. Notice the green circle showing topological features that born and die at the same time.

The 0-persistence barcode (i.e., the lifetime of connected components) and the 1-persistence barcode (i.e., the lifetime of tunnels) are explored according to a uniform sampling. More precisely, given a positive integer $n$ (being $n=24$ in our experimental results, obtained by cross validation), we compute the integer $h=\left\lfloor\frac{k}{n}\right\rfloor$, which represents the width of the "window" we use to analyze the persistence barcode, being $k$ the biggest distance of a vertex in $\partial K(I)$ to the given plane $\pi$. Later, for $s=0, \ldots, n-1$, the 0 - (resp. 1-) persistence barcode of $K_{\pi}$ is sampled to obtain:

(a) Number of 0- (resp. 1-) homology classes that are born before $s \cdot h$ and persist or die after $s \cdot h$. 

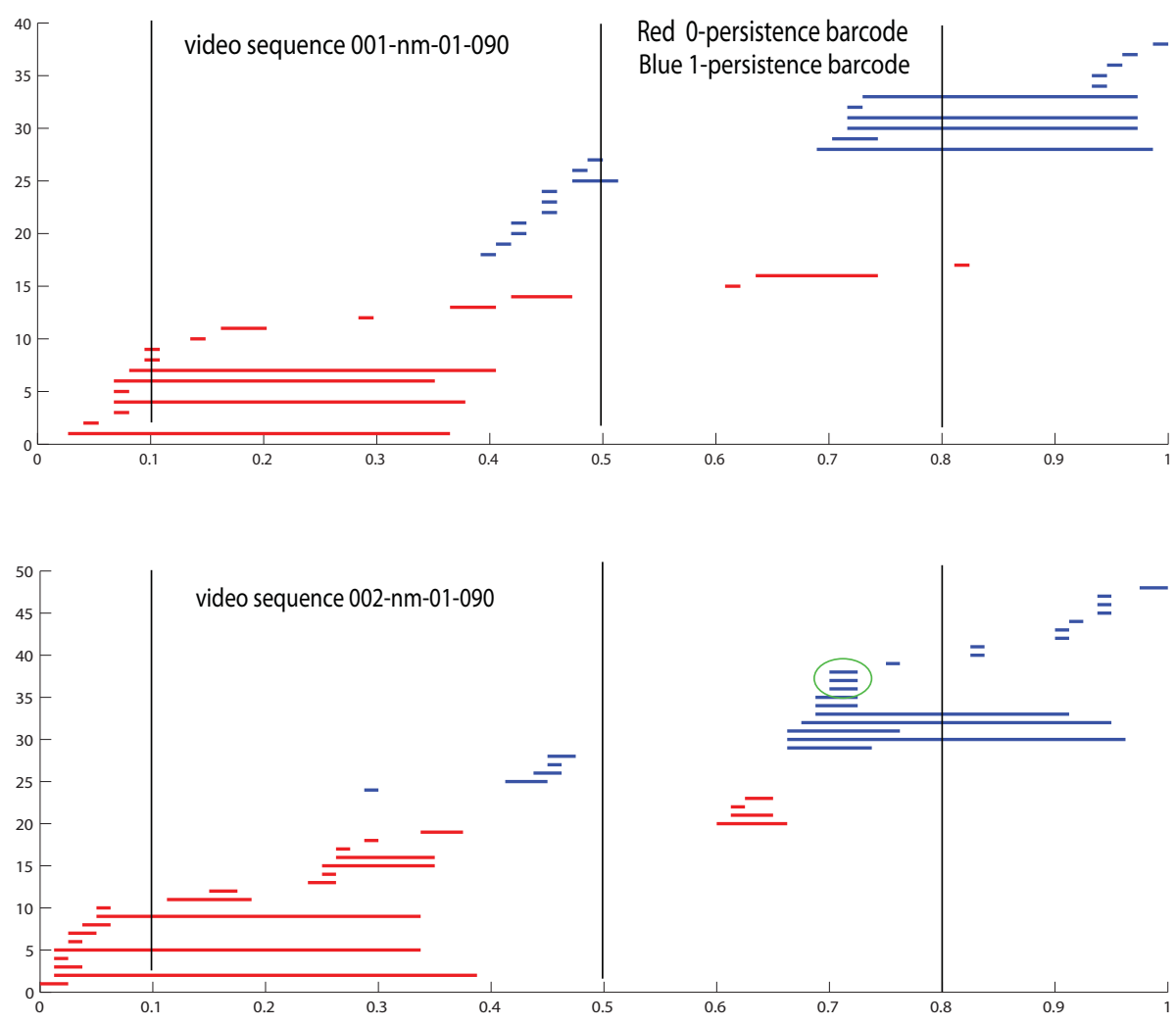

Fig. 5. Persistence barcodes for the sequences 001-nm-01-090 and 002-nm-01-090 of CAISA-B dataset. Horizontal axis represents the distance to the reference plane, normalized to $[0.1]$.

(b) Number of 0- (resp. 1-) homology classes that are born in $s \cdot h$ or later, and before $(s+1) \cdot h$.

A vector of $2 n$ entries is then formed containing (a) in entry $2 s$, for $0<s \leq n$; and (b) in entry $2 s+1$, for $0 \leq s<n$.

For example, suppose that a $j$-homology class is born and dies within the interval $[s \cdot h,(s+1) \cdot h)$. Then, in this case, we add 1 in entry $2 s+1$. On the other hand, suppose that a $j$-homology class is born in the interval $[s \cdot h,(s+1) \cdot h)$ and dies in $[t \cdot h,(t+1) \cdot h)$ for some $t, s<t \leq n$. Then, in this case, we add 1 in entry $2 s+1$, and in entries $2 j$ for $s<j \leq t$.

Dividing the entries in two categories (a) and (b), small details in the object are highlighted, which is crucial for distinguishing two different gaits. For example, let us suppose an scenario in which $m j$-homology classes are born in $[s$. $h,(s+1) \cdot h)$ and persist or die at the end of $[(s+1) \cdot h,(s+2) \cdot h)$ and not any other $j$-homology class is born, persists or dies in these intervals. Then, we put 0 in entries $2 s$ and $2 s+3$, and $m$ in entries $2 s+1$ and $2 s+2$. On the other hand, let us suppose that $m j$-homology classes are born and die in $[s \cdot h,(s+1) \cdot h)$ and in $[(s+1) \cdot h,(s+2) \cdot h)$ and not any other $j$-homology class is born, persists or dies in these intervals. Then, we put 0 in entries $2 s$ and $2 s+2$ and $m$ in entries $2 s+1$ and $2 s+3$. Observe that only considering (a) and (b) separately, we can distinguish both scenarios.

This way, fixed a plane $\pi$, we obtain two $2 n$-dimensional vectors for $K_{\pi}$, one for the 0 -persistence barcode and the other for the 1-persistence barcode associated to the filtration $K_{\pi}$.
Since we have eight planes, $\left\{\pi_{1}, \ldots \pi_{8}\right\}$, and two vectors per plane, $\left\{\mathcal{V}_{\pi_{i}}^{0}, \mathcal{V}_{\pi_{i}}^{1}\right\}_{i=1, \ldots, 8}$, we have a total of sixteen $2 n$ dimensional vectors which form the topological signature for a gait sequence.

For comparing the topological signatures of two sequences, we add up the angle between each pair of the corresponding vectors conforming the topological signatures. Since a signature consists of sixteen vectors, the best comparison for two sequences is obtained when the total sum is zero and the worst is $90 \cdot 16=1440$. Observe that in our previous papers [3], [8], [9], [10], we used the cosine distance to compare two given topological signatures. We have noticed that using the angle instead of the cosine, the efficiency increases by $5 \%$.

Notice that, in the case the gait sequence contains more than a gait cycle, the module of the vectors $\left\{\mathcal{V}_{\pi_{i}}^{0}, \mathcal{V}_{\pi_{i}}^{1}\right\}_{i=1, \ldots, 8}$ can increase with respect to the gait sequence that contains exactly a gait cycle, but the direction remains nearly the same. This way, using the angle between two vectors as dissimilarity measure, we do not have to take care about the length of a gait sequence used to compute the topological signature. This is not true if we consider the classical bottleneck distance for comparing two different persistence diagrams (i.e. barcodes) [11]. 


\section{EXPERIMENTAL RESUlTS}

In this section we show the accuracy results in two experiments using CASIA-B dataset ${ }^{2}$. The CASIA-B dataset has 124 persons, and 10 samples for each of the 11 different angles at which a person is taken. For each angle there are six samples walking under natural conditions, which means without carrying a bag or wearing a coat (CASIA-Bnm), there are two samples of persons carrying some sort of bag (CASIA$\mathrm{Bbg}$ ) and the remaining two samples for persons wearing coat (CASIA-Bcl). CASIA-B dataset provides image sequences with background segmentation for each person.

In the first experiment we used four sequences by person from CASIA-Bnm dataset to train. We used the other two sequences by person from CASIA-Bnm and the sequences from CASIA-Bbg and CASIA-Bcl to test. Our results for lateral view (90 degrees) are shown in Table I, where we take the cross validation average $\left(\left(\begin{array}{l}6 \\ 4\end{array}\right)=15\right.$ combinations) of accuracy at rank 1 from the candidates list. The result of our previous method [3] was also evaluated using the lowest fourth part of the body silhouette.

In the second experiment, we followed the protocol used in [5, Section 5.3]. This way, we considered a mixture of normal, carrying-bag and wearing-coat sequences, since it models a more realistic situation where persons do not collaborate while the samples are being taken. Specifically, six sequences were used to training (four normal sequences, one carrying-bag sequence and one wearing-coat sequence), the rest was used to test. Table II shows the result of the accuracy.

A third experiment was carried out for obtaining Fig. 6. In this case six sequences were used for training (one with the person carrying a bag, one with the person wearing a coat and four with the person walking under natural conditions). Using this training data we generated 123 topological signatures, one for each person in the database. We used the remaining sequence of the person carrying a bag and the one wearing a coat for testing. This gave us 246 sequences for testing: 123 persons times 2 sequences by person. We must point out that person labeled as 005 in CAISA-B was removed from the experiment due to poor quality.

Using the obtained topological signatures and testing set we obtained:

(1) The set of all possible comparisons between the obtained signatures and the signatures of the test sequences, corresponding to the same person. This set is called True Positive (TP) and contains 246 comparison values.

(2) The set of all possible comparisons between the obtained signatures and the signatures of the test sequences, corresponding to different persons. This set is called True Negative (TN) and contains $123 * 244=30012$ values.

For obtaining Fig. 6, we first restricted TN to its 246 smallest values, in order to balance the sets TP and TN. Then we represented in the $y$ axis the percent of values in the TP set lower than a threshold as a red curve and the same for the TN

\footnotetext{
${ }^{2}$ http://www.cbsr.ia.ac.cn/GaitDatasetB-silh.zip
}

set as a blue curve. The $x$ axis represents all the considered thresholds. For example, $92.6 \%$ of the data in the set TP are values smaller than 253.8 , since the red curve shows that for $x=253.8$ we have that $y=92.6 \%$.

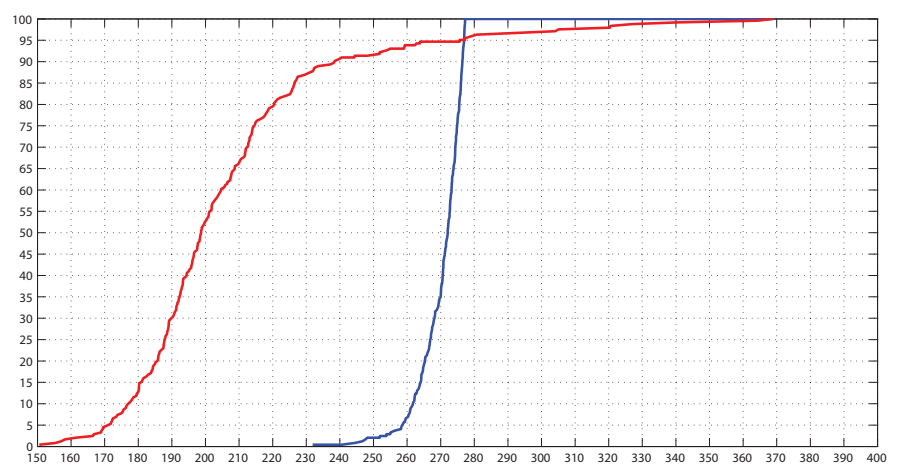

Fig. 6. Result of the comparison between the training set and the test set for the topological signatures of the sequences representing the same person (in red) and the one between the training set and the test set for the topological signatures of the sequences representing different persons (in blue).

More examples and the source code written in Matlab can be obtained visiting the web page: http://grupo.us.es/cimagroup/.

\section{ANALYSIS OF THE RESUlTS}

In Table I we see that the best result of our method was for the set of normal sequences (CASIA-Bnm) and the worst was for the set of persons carrying bags. This is due to that bags can affect the accuracy of the method (see the lowest fourth part of the body silhouette in Fig. 7). Moreover, the weight of the bag can change the dynamic of the gait. On the other hand, the features obtained from the lowest fourth part of the body silhouette gave an accuracy for the normal sequences of $94.1 \%$, which only decreases $3.9 \%$ with respect to our previous paper [3] using the whole body silhouette (98.0\%). This confirms that the highest information in the gait is in the motion of the legs, which supports the results given in [5].

Nevertheless, as we can see in Tables I and II, our method outperforms previous methods for gait recognition with or without carrying a bag or wearing a coat. Furthermore, we show in Table I that the changes introduced to obtain our new method derive in an improvement with respect to our previous solution [3].

Besides, the algorithm explained in [5] decreases considerably the accuracy obtained by training mixing the normal, carrying-bag and wearing-coat sequences (see Table II). On the contrary, our algorithm improves the accuracy for the whole test set. Comparing the two tables, we can as well arrive to the conclusion that training with more heterogeneous data gives to our method a more powerful representation for the classification step and in that case, our method outperforms in more that $35 \%$ the results given in [5].

Finally, the results presented in this paper show the power of the Theory of Persistent Homology to obtain the structural features of the dynamic of the legs. 
TABLE I

ACCURACY (IN \%) USING TRAINING SETS CONSISTING OF SAMPLES UNDER SIMILAR COVARIATE CONDITIONS (WITHOUT CARRYING A BAG OR WEARING A COAT).

\begin{tabular}{|c|c|c|c|c|}
\hline Methods & CASIA-Bbg & CASIA-Bcl & CASIA-Bnm & Average \\
\hline Tieniu.T [1] & 52.0 & 32.73 & 97.6 & 60.8 \\
\hline Khalid.B [5] & 78.3 & 44.0 & 100 & 74.1 \\
\hline Singh.S [13] & 74.58 & 77.04 & 93.44 & 81.7 \\
\hline Previous Method [3] & 75.8 & 75.45 & 90.3 & 80.5 \\
\hline Our Method & 84.2 & 87.6 & 94.1 & 88.6 \\
\hline
\end{tabular}

TABLE II

ACCURACY (IN \%) USING TRAINING SETS CONSISTING OF SAMPLES UNDER DIFFERENT COVARIATE CONDITIONS (WALKING-NORMAL, CARRYING-BAG AND WEARING-COAT)

\begin{tabular}{ccccc}
\hline Methods & CASIA-Bbg & CASIA-Bcl & CASIA-Bnm & Average \\
\hline Khalid.B [5] & 55.6 & 34.7 & 69.1 & 53.1 \\
Our Method & $\mathbf{9 2 . 3}$ & $\mathbf{9 4 . 3}$ & $\mathbf{9 4 . 7}$ & $\mathbf{9 3 . 8}$ \\
\hline
\end{tabular}

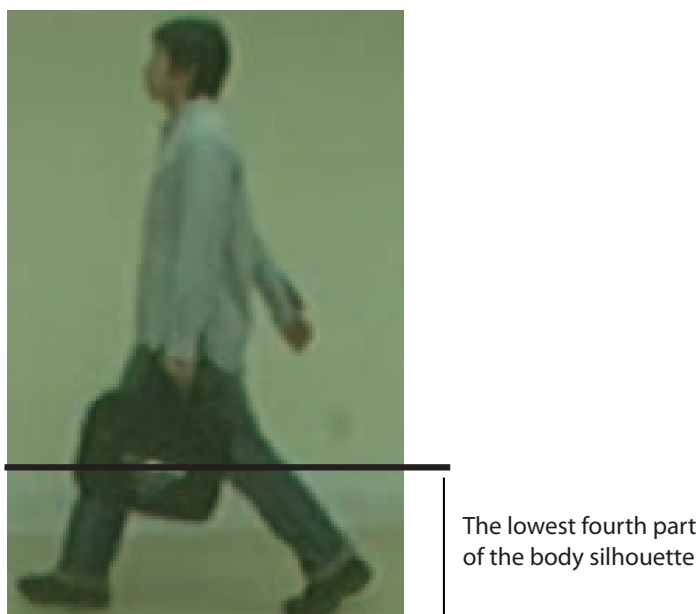

Fig. 7. Lowest four part of the body occluded by a bag.

\section{CONCLUSION}

In this paper we have presented an algorithm for gait recognition, a technique with special attention in tasks of video surveillance. We have used persistent homology to model the gait, similar as we did in our previous approaches, although the algorithm presented here is slightly different to our previous ones in a way that it is more robust to variations in the amount of simplices considered and removes as well any dependency with respect to the stability of the sorting algorithm used for obtaining the filtration. Besides, the topological features have been tested here using only the lowest fourth part of the body silhouette. Then, the effects of variations unrelated to the gait in the upper body part, which are very frequent in real scenarios, decrease considerably. Finally, the results presented in the paper improve considerably the accuracy in the state of the art.

Acknowledgments: This work has been partially supported by MINECO/FEDER-UE under grant MTM2015-67072-P. We thank anonymous referees for their valuable comments and suggestions.

\section{REFERENCES}

[1] S. Yu, D. Tan, and T. Tan, "A framework for evaluating the effect of view angle, clothing and carrying condition on gait recognition," in Pattern Recognition, 2006. ICPR 2006. 18th International Conference on, vol. 4. IEEE, 2006, pp. 441-444.

[2] C. P. Lee, A. W. Tan, and S. C. Tan, "Time-sliced averaged motion history image for gait recognition," Journal of Visual Communication and Image Representation, vol. 25, no. 5, pp. 822-826, 2014.

[3] J. Lamar-Leon, E. Garcia-Reyes, and R. Gonzalez-Diaz, "Human gait identification using persistent homology," in Progress in Pattern Recognition, Image Analysis, Computer Vision, and Applications 17th Iberoamerican Congress, CIARP 2012, Buenos Aires, Argentina, September 3-6, 2012. Proceedings, 2012, pp. 244-251.

[4] Y. Zhang, S. Jiang, Z. Yang, Y. Zhao, and T. Guo, "A score level fusion framework for gait-based human recognition," in Multimedia Signal Processing (MMSP), 2013 IEEE 15th International Workshop on. IEEE, 2013, pp. 189-194.

[5] K. Bashir, T. Xiang, and S. Gong, "Gait recognition without subject cooperation," Pattern Recognition Letters, vol. 31, no. 13, pp. 2052 2060, 2010.

[6] C. Chen, J. Liang, H. Zhao, H. Hu, and J. Tian, "Frame difference energy image for gait recognition with incomplete silhouettes," Pattern Recognition Letters, vol. 30, no. 11, pp. 977-984, 2009.

[7] D. A. Winter, Biomechanics and motor control of human gait: normal, elderly and pathological, 1991.

[8] J. Lamar-Leon, A. Cerri, E. Garia-Reyes, and R. Gonzalez-Diaz, "Gaitbased gender classification using persistent homology," in Progress in Pattern Recognition, Image Analysis, Computer Vision, and Applications - 18th Iberoamerican Congress, CIARP 2013, Havana, Cuba, November 20-23, 2013, Proceedings, Part II, 2013, pp. 366-373.

[9] J. Lamar-Leon, R. Alonso-Baryolo, E. Garcia-Reyes, and R. GonzalezDiaz, "Gait-based carried object detection using persistent homology," in Progress in Pattern Recognition, Image Analysis, Computer Vision, and Applications - 19th Iberoamerican Congress, CIARP 2014, Puerto Vallarta, Mexico, November 2-5, 2014. Proceedings, 2014, pp. 836-843.

[10] _ " "Topological features for monitoring human activities at distance," in Activity Monitoring by Multiple Distributed Sensing - Second International Workshop, AMMDS 2014, Stockholm, Sweden, August 24, 2014, 2014, pp. 40-51.

[11] H. Edelsbrunner and J. Harer, Computational topology: an introduction. American Mathematical Soc., 2010.

[12] R. Ghrist, "Barcodes: The persistent topology of data," Bull. Amer. Math. Soc. 45 (2008), 61-75, vol. 45, pp. 61-75, 2008.

[13] S. Singh and K. Biswas, "Biometric gait recognition with carrying and clothing variants," in Pattern Recognition and Machine Intelligence. Springer, 2009, pp. 446-451. 